\title{
Committee Listing
}

Published online: 8 January 2018

(C) International Association of Medical Science Educators 2018

Thank you to all who helped make the 2017 IAMSE meeting a huge success!

\section{Program Committee}

Kathryn Huggett (Program Chair)

Michael Bradbury

Colleen Croniger

Stephen Everse

Alice Fornari

Mark Hernandez

Peter de Jong (Review Committee

Chair)

Veronica Michaelsen (IAMSE President)

Luke Mortensen (2016 Program Chair)

James Pickering

Kelly Quesnelle

Greg Smith

Amy Wilson-Delfosse

Rick Vari (2018 Program Chair)

Jonathan Wisco

Nikki Woods

Stephanie Wragg

\section{Review Committee}

Peter de Jong (Chair)

Francis Achike

Reid Adams

Marta Ambrozewicz

Peter Anderson

Mark Andrews

Michael Bradbury

Judith Brenner

Era Buck

Raeann Carrier

Rob Carroll

Craig Clarkson

Maurice Clifton
Mark Clunes
Bonny Dickinson
Nyla Dil
Isabel Dominguez
Lisa Elferink
Leslie Ellis
Carrie Elzie
Alice Fornari
Thomas Gaddy
Nathalie Garcia-Russell
Laurel Gorman
Bonnie Granat
Colin Greengrass
Zeynep Gromley
Inaya Hajj Hussein
Adi Haramati
Mark Hernandez
Kerstin Honer zu Bentrup
Brady Janes
Christine Kell
Claire Kirchhoff
Floyd Knoop
Valeriy Kozmenko
Steven Kubalak
Jan Kuks
Jennifer Li
Janet Lindsley
Michelle Linsenmeyer
Kelly McCarthy
Maureen McEnery
Jennifer Meka
Misa Mi
Sarah Morley
Sabyasachi Moulik

Brian Neubauer

Khiet Ngo

Carmen Obando

Neil Osheroff

Anthony Paganini

Gustavo Patino

Susan Perlis

Thomas Peterson

Kevin Phelan

Thomas Pisarri

Amy Prunuske

Dale Quest

Jayne Reuben

Ellen Robertson

Michael Robinson

Gary Rosenfeld

Amina Sadik

Norma Saks

Dawn Schocken

Michiel Schokking

Patricia Sexton

Leah Sheridan

Paula Smith

Greg Smith

Abi Sriharan

Tracey Taylor

Julie Tebo

Wezenet Tewodros

Nitsa Topale

Virginia Uhley

Richard Vari

Nagaswami Vasan

Audrey Vasauskas

Dan Webster

Jonathan Wisco 